\title{
Harmonic numbers and finite groups
}

\author{
Sekhar Jyoti Baishya (*) - Ashish Kumar Das (*)
}

ABstract - Given a finite group $G$, let $\tau(G)$ be the number of normal subgroups of $G$ and $\sigma(G)$ be the sum of the orders of the normal subgroups of $G$. The group $G$ is said to be harmonic if $H(G):=|G| \tau(G) / \sigma(G)$ is an integer. In this paper, all finite groups for which $1 \leq H(G) \leq 2$ have been characterized. Harmonic groups of order $p q$ and of order $p q r$, where $p<q<r$ are primes, are also classified. Moreover, it has been shown that if $G$ is harmonic and $G \neq C_{6}$, then $\tau(G) \geq 6$.

Mathematics SubJeCt Classification (2010). 11A25, 20D60, $20 \mathrm{E} 99$.

KEYwoRDs. Finite groups, harmonic numbers, harmonic groups.

\section{Introduction}

Given a positive integer $n$, let $H(n)$ denote the harmonic mean of all positive divisors of $n$. Thus, $H(n)=n \tau(n) / \sigma(n)$, where $\tau(n)$ and $\sigma(n)$ are respectively the number and sum of all positive divisors of $n$. A positive integer $n$ is said to be harmonic if $H(n)$ is an integer. 1 is the only odd harmonic number known so far. Harmonic numbers were first studied by Oystein Ore [11] in 1948, and they attracted the attention of many others (see, for example, [1], [3], [4], [5], [6], etc.) primarily because of their connection with perfect numbers. It may be recalled here that a positive integer $n$ is said to be perfect if $\sigma(n)=2 n$, and it is easy to show that every perfect number is harmonic (see [11]); however, 140 is harmonic but not perfect.

In 2001, T. Leinster [8] developed and studied a group-theoretic analogue of perfect numbers. A finite group is said to be a perfect

(*) Indirizzo degli A.: Department of Mathematics, North-Eastern Hill University, Permanent Campus, Shillong-793022, Meghalaya, India.

E-mail: sekharnehu@yahoo.com_akdasnehu@gmail.com 
group or an immaculate group or a Leinster group if the sum of the orders of its normal subgroups equals twice the order of the group itself. Clearly, a finite cyclic group $C_{n}$ is a Leinster group if and only if its order $n$ is a perfect number. In fact, the abelian Leinster groups are precisely the finite cyclic groups whose orders are perfect numbers. More information on this and the related concepts can be found in the works of A. K. Das [2], M. Tărnăuceanu [13, 9], T. De Medts and A. Maróti [10], etc.

In this paper we initiate a study of a group-theoretic analogue of harmonic numbers.

\section{Definition and basic results}

Given a finite group $G$, let us write $H(G)=|G| \tau(G) / \sigma(G)$, where $\tau(G)$ is the number of normal subgroups of $G$ and $\sigma(G)$ is the sum of the orders of all normal subgroups of $G$, that is, $\tau(G)=\sum_{N \unlhd G} 1$ and $\sigma(G)=\sum_{N \unlhd G}|N|$. Note that $H(G)=1$ if and only if $G$ is trivial. In fact, $\sigma(G)>\tau(G)>H(G)>1$ whenever $G$ is a finite non-trivial group. Also, if two finite groups $G_{1}$ and $G_{2}$ are coprime in the sense of [2], then we have $\tau\left(G_{1} \times G_{2}\right)=\tau\left(G_{1}\right) \tau\left(G_{2}\right)$ and $\sigma\left(G_{1} \times G_{2}\right)=\sigma\left(G_{1}\right) \sigma\left(G_{2}\right)$, and so, $H\left(G_{1} \times G_{2}\right)=H\left(G_{1}\right) H\left(G_{2}\right)$.

Definition 2.1. A finite group $G$ is said to be a harmonic group if $H(G)$ is an integer.

It is easy to see that a finite cyclic group $C_{n}$ is harmonic if and only if its order $n$ is a harmonic number. Therefore, since 6 is the only harmonic number which is square-free (see [4]) and since the finite abelian groups of square-free order are all cyclic, it follows that $C_{6}$ is the only abelian harmonic group whose order is square-free. Note that $C_{6}$ is also a Leinster group. However, in general, a harmonic group need not be Leinster, and a Leinster group need not be harmonic. For example, using GAP [15], one can see that the groups $C_{4} \times S_{3}, C_{3} \times C_{16}, C_{2} \times C_{4} \times S_{3}$ are harmonic but not Leinster, and the groups $C_{3} \times C_{4}, C_{7} \times C_{8}$ are Leinster but not harmonic. The following small result helps in determining whether a given Leinster group is harmonic or not.

Proposition 2.2. Let $G$ be a Leinster group. Then $G$ is harmonic if and only if $\tau(G)$ is even. 
Proof. Since $G$ is a Leinster group, we have $\sigma(G)=2|G|$, and so, $H(G)=\tau(G) / 2$. Thus, $H(G)$ is an integer if and only if $\tau(G)$ is even.

Note that if $G$ is a Leinster group of odd order, then $\tau(G) \equiv$ $\sigma(G) \equiv 0 \bmod 2$. Therefore, in view of Proposition 2.2, every Leinster group of odd order is harmonic. It may be mentioned here that only one Leinster group of odd order could be found so far, namely, $\left(C_{127} \times C_{7}\right) \times$ $C_{3^{4} .11^{2} .19^{2} .113}$, and it was first discovered by F. Brunault [14]. In [10, Proposition 2.2], all Leinster groups of the form $\langle x, y| x^{n}=y^{2^{t}}=1$, $\left.y^{-1} x y=x^{-1}\right\rangle$, where $n$ is an odd positive integer and $t$ is positive integer, have been classified; however, in view of Proposition 2.2, none of these Leinster groups are harmonic.

Recall that a non-trivial group is said to be simple if it has no proper non-trivial normal subgroup. The following result shows that no finite simple group is harmonic.

Proposition 2.3. If $G$ is a finite simple group, then $1<H(G)<2$.

Proof. Since $\{1\}$ and $G$ are the only normal subgroups of $G$, we have $H(G)=2|G| /(1+|G|)$. Hence, the result follows.

A group is said to be semi-simple if it is a direct product of non-abelian simple groups. In this connection, we have the following result.

Proposition 2.4. No finite semi-simple group is harmonic.

Proof. Let $G$ be a finite semi-simple group. Then $G=H_{1} \times$ $H_{2} \times \cdots \times H_{n}$, where each $H_{i}(1 \leq i \leq n)$ is a finite non-abelian simple group. Therefore, we have $\tau(G)=\prod_{i} \tau\left(H_{i}\right)=2^{n}$ and $\sigma(G)=\prod_{i} \sigma\left(H_{i}\right)=$ $\prod_{i}\left(1+\left|H_{i}\right|\right)$. Clearly, $\sigma(G)$ is odd because all finite non-abelian simple groups are of even order. Thus, if $G$ is harmonic, it follows from the definition of $H(G)$ that $\sigma(G)$ divides $|G|$, which is impossible as $\sigma(G)>|G|$. Hence $G$ is not harmonic.

Continuing with the finite groups that are not harmonic, we also have the following result on $p$-groups.

Proposition 2.5. If $G$ is a finite group with $|G|=p^{r}$, where $p$ is a prime and $r \geq 1$, then $G$ is not harmonic. 
Proof. Since $\operatorname{ged}(p, \sigma(G))=1$ and $\tau(G)<\sigma(G)$, it follows from the definition of $H(G)$ that $G$ is not a harmonic group.

Given a finite non-trivial group $G$, the following result gives the best possible lower bounds (depending on whether $G$ is simple or not) for the values of $H(G)$. In particular the result says that there is no finite group $G$ with $1<H(G)<4 / 3$.

Proposition 2.6. Let $G$ be a finite non-trivial group. If $G$ is simple, then $H(G) \geq 4 / 3$, where the equality holds if and only if $G \cong C_{2}$. If $G$ is not simple, then $H(G) \geq 12 / 7$, where the equality holds if and only if $G \cong C_{4}$.

Proof. If $G$ is simple, then it is easy to see that $H(G)=$ $2|G| /(1+|G|) \geq 4 / 3$, where the equality holds if and only if $|G|=2$. Let us now assume that $G$ is not a simple group. Then $\tau(G) \geq 3,|G| \geq 4$, and for every proper normal subgroup $N$ of $G$ we have $|N| \leq|G| / 2$. Therefore, it follows that

$$
H(G) \geq \frac{|G| \tau(G)}{1+|G|+(\tau(G)-2)|G| / 2} \geq \frac{12}{7} .
$$

Now, for $G \neq C_{4}$, we have $\tau(G)>3$ or $|G|>4$, and so it follows from (1) that $H(G)>12 / 7$. On the other hand, it is easy to see that $H\left(C_{4}\right)=12 / 7$. This completes the proof.

\section{Finite groups with $H(G) \leq 2$}

Given a finite group $G$, we have seen in the previous section that $H(G) \geq 1$, where the equality holds if and only if $G$ is trivial. In this section we characterize all finite groups that satisfy the condition $1<H(G) \leq 2$.

Proposition 3.1. Let $G$ be a finite non-trivial group. Then $H(G) \leq 2$ if and only if one of the following assertions holds:

(a) $G$ is simple.

(b) $G \cong C_{6}$.

(c) Every proper non-trivial normal subgroup of $G$ is of index 2 . 
Proof. Note that $H(G)<2$ if $G$ is simple, and that $H\left(C_{6}\right)=2$. Also, if every proper non-trivial normal subgroup of $G$ is of index 2, then $2 \sigma(G)=2(1+|G|+(\tau(G)-2)|G| / 2)>\tau(G)|G|$, and so, $H(G)<2$.

Conversely, suppose that $H(G) \leq 2$ and that none of the given assertions holds. Then $|G| \geq 8$ and $2 \sigma(G) \leq 2(1+|G|+(\tau(G)-3)|G| / 2+|G| / 3)<$ $|G| \tau(G)$, that is, $H(G)>2$. This contradiction completes the proof.

As an immediate consequence, we have the following corollary.

COROLlary 3.2. Let $G$ be a finite group. Then $H(G)=2$ if and only if $G \cong C_{6}$.

PRoof. The result follows from the proof of Proposition 3.1.

A group $G$ is said to be an extension of a normal subgroup $N$ by a group $H$ if $G / N \cong H ; G$ is said to be a trivial extension of $N$ by $H$ if $G \cong H \times N$. We use this concept to characterize all finite non-trivial groups that satisfy the condition $H(G)<2$.

THEOREM 3.3. Let $G$ be a finite group and $G^{\prime}$ be its commutator subgroup. Then $1<H(G)<2$ if and only if one of the following assertions holds:

(a) $G$ is a simple group.

(b) $G \cong C_{2} \times C_{2}$.

(c) $G \cong C_{4}$.

(d) $G^{\prime}$ is a simple group, and $G$ is a non-trivial extension of $G^{\prime}$ by $C_{2}$.

(e) $G^{\prime} \cong T \times T$, where $T$ is a non-abelian simple group, and $G$ is an extension of $G^{\prime}$ by $C_{2}$ such that the proper non-trivial normal subgroups of $G^{\prime}$ are conjugate in $G$.

Proof. If any one of the assertions (a), (b) and (c) holds, then it is easy to see that $1<H(G)<2$. On the other hand, if the assertion (d) or (e) holds, then it follows that $G^{\prime}$ is the only proper non-trivial normal subgroup of $G$, and hence, $H(G)=3|G| /\left(1+\left|G^{\prime}\right|+|G|\right)=6|G| /(2+3|G|)<2$.

Conversely, suppose that $1<H(G)<2$ and that $G$ is not simple. Then, by Proposition 3.1 and Corollary 3.2, every proper non-trivial normal subgroup of $G$ is of index 2. If $G$ is abelian, then $|G|=4$, and so, $G \cong C_{2} \times C_{2}$ or $G \cong C_{4}$. So, we may assume that $G$ is non-abelian. In that case, $G^{\prime}$ is the only proper non-trivial normal subgroup of $G$ and $\left|G: G^{\prime}\right|=2$. Thus, it follows that $G$ is a non-trivial extension of $G^{\prime}$ by $C_{2}$. 
Now, by [7, 1.7.4 and 1.7.5], we have either $G^{\prime} \cong\left(C_{p}\right)^{m}$ or $G^{\prime} \cong(T)^{n}$, where $m$ and $n$ are positive integers, $p$ is a prime, and $T$ is a non-abelian simple group. In fact, $p$ is an odd prime, because a non-abelian 2-group has more than one proper non-trivial normal subgroup. If $G^{\prime} \cong C_{p}$, or if $G^{\prime} \cong T$, then we are done. If $G^{\prime} \cong\left(C_{p}\right)^{m}$ with $m>1$, then the conjugate action of $G$ on $G^{\prime}$ induces an action of $C_{2}$ on $\left(C_{p}\right)^{m}$ through $\operatorname{Aut}\left(G^{\prime}\right) \cong \mathrm{GL}(m, p)$. Since no proper non-trivial normal subgroup of $G^{\prime}$ is normal in $G$, this action is irreducible. Therefore, by [12, Theorem 2.3.2], 2|( $\left.p^{m}-1\right)$ and $2 \nmid\left(p^{d}-1\right)$ for any positive integer $d<m$, which is impossible. Hence, $G^{\prime} \nsucceq\left(C_{p}\right)^{m}$ with $m>1$. On the other hand, if $G^{\prime} \cong(T)^{n}$ with $n>1$, then $n=2$ and the proper non-trivial normal subgroups of $G^{\prime}$ are conjugate in $G$. This is because the minimal normal subgroups of $G^{\prime}$ are conjugate in $G$ (see [7, 1.7.5]), and each of these subgroups has exactly two conjugates in $G$ (the normalizers of these subgroups in $G$ being $G^{\prime}$ ). This completes the proof.

As an immediate consequence we have the following corollary.

Corollary 3.4. Let $G$ be a finite solvable group. Then $1<H(G)<2$ if and only if $G \cong C_{2}, C_{2} \times C_{2}, C_{4}, C_{p}$ or $D_{2 p}$, where $p$ is an odd prime.

\section{Harmonic groups with $\tau(G) \leq 5$}

In this section we determine whether or not a finite group $G$ with $\tau(G) \leq 5$ is harmonic. Our first result gives a necessary condition for a finite non-trivial group to be harmonic.

Proposition 4.1. Let $G$ be a non-trivial harmonic group. Then $\tau(G) \geq 4$, where the equality holds if and only if $G \cong C_{6}$.

Proof. We have $H(G) \geq 2$. If $H(G)=2$, then, by Corollary 3.2, $G \cong C_{6}$, and hence, $\tau(G)=4$. If $H(G) \geq 3$, then $\tau(G) \geq H(G)+1 \geq 4$. Therefore, it remains to show that $G \cong C_{6}$ if $\tau(G)=4$, and for this it is enough to show that there is no harmonic group $G$ with $H(G)=3$ and $\tau(G)=4$. On the contrary, suppose that $G$ is a harmonic group with $H(G)=3$ and $\tau(G)=4$. Let $M$ and $N$ denote the two proper non-trivial normal subgroups of $G$. Let $|M|=m$ and $|N|=n$. Then, from the definition of $H(G)$, we have

$$
3(1+m+n)=|G| .
$$


By the choice of $G$, we have only two possibilities to examine, namely, $M \subsetneq N$ and $M \cap N=\{1\}$. If $M \subsetneq N$, then it follows from (2) that $m=3, n=6$ or 12 , and accordingly, $|G|=30$ or 48 . But a group of order 30 has a normal subgroup of order 5 , and a group of order 48 does not have a maximal normal subgroup of order 12 . Therefore, it is not possible to have $M \subsetneq N$. If $M \cap N=\{1\}$, then we have $G=M N$, and so (2) becomes

$$
3(1+m+n)=m n \text {. }
$$

It follows that $3 \mid m$ or $3 \mid n$. Without any loss we may assume that $3 \mid m$. Then, by (3), $(n-3) \mid(n+1)$, and so we have $n=4,5$ or 7 . Since $G / M$ is simple, $n=|G / M| \neq 4$. If $n=5$ or 7 , then, by (3), we have $m=9$ or 6 , none of which is possible as $m$ is the order of the simple group $G / N$. Hence, it is impossible to have $M \cap N=\{1\}$ either. Thus, it follows that if $G$ is a harmonic group with $\tau(G)=4$, then we have $H(G)=2$, and so, $G \cong C_{6}$.

As a consequence we have the following result.

COROLlaRY 4.2. $\quad$ Let $G$ be a finite group satisfying one of the following conditions:

(a) $G \cong H \times K$, where $H$ and $K$ are simple groups.

(b) $|G|=p q$, where $p$ and $q$ are primes.

Then $G$ is a harmonic group if and only if $G \cong C_{6}$.

Proof. Let $G$ be a harmonic group satisfying one of the given conditions. Since $p$-groups are not harmonic (by Proposition 2.5), the simple groups $H$ and $K$ (if (a) holds) are almost coprime in the sense of [2], and the primes $p$ and $q$ (if (b) holds) are distinct. Thus, if (a) is satisfied, then $\tau(G)=\tau(H) \tau(K)=4$, and so, $G \cong C_{6}$ (by Proposition 4.1). On the other hand, if (b) is satisfied, then $G$ is abelian (otherwise, $\tau(G)=3$ ); in fact, $G \cong C_{p} \times C_{q}$, that is, $G$ satisfies (a), and so we have $G \cong C_{6}$ once again. This completes the proof as $C_{6}$ is already seen to be harmonic.

Remark 4.3. Using Proposition 4.1, one may also conclude at once that the symmetric group $S_{n}$ and the alternating group $A_{n}$ are not harmonic for any integer $n \geq 2$.

The following proposition says that there is no harmonic group with $\tau(G)=5$. 
Proposition 4.4. If $G$ is a finite group with $\tau(G)=5$, then $G$ is not harmonic.

Proof. Using GAP [15], or otherwise, one can see that there is no harmonic group, which has exactly five normal subgroups and whose order is $60,90,300,500$ or 600 . Let us assume that $G$ is a harmonic group with $\tau(G)=5$ and $|G| \notin\{60,90,300,500,600\}$. Then, in view of Corollary $4.2, G$ is indecomposable; noting that if $G$ is a direct product of two groups at least one of which is non-simple, then we have $\tau(G) \geq 6$. Let $N_{1}, N_{2}, N_{3}$ be the three distinct proper non-trivial normal subgroups of $G$, and $n_{1}, n_{2}, n_{3}$ denote their order. Note that each $n_{i}$ is of the form $|G| / n$, where $n \geq 2$. Without any loss, we may assume that $n_{1} \leq n_{2} \leq n_{3}$. Then, $N_{1}$ is a minimal normal subgroup, and $N_{3}$ is a maximal normal subgroup. Note that we always have $N_{1} \subsetneq N_{3}$, otherwise $G \cong N_{1} \times N_{3}$. Also, we never have $N_{1} \nsubseteq N_{2} \nsubseteq N_{3}$, otherwise $G \cong N_{2} \times N_{3}$. Thus, there are three possibilities, namely, (A) $N_{1} \varsubsetneqq N_{2} \subsetneq N_{3}$, (B) $N_{1}=N_{2} \cap N_{3}$ with $N_{2} N_{3}=G$, and (C) $N_{1} N_{2}=N_{3}$ with $N_{1} \cap N_{2}=\{1\}$.

In view of certain standard results from the theory of finite groups, it is easy to see that the following assertions hold:

(a) $n_{1}$ is a power of a prime whenever $n_{1}<60$.

(b) $n_{1}$ is a proper divisor of $n_{3}$.

(c) $|G| / n_{3}$ is a prime whenever $|G| / n_{3}<60$.

(d) If $n_{2} \nmid n_{3}$, then $n_{1}$ is a proper divisor of $n_{2}$; in fact, $n_{2}=n_{1}|G| / n_{3}$.

(e) $\tau\left(G / N_{2}\right) \leq 3$, and so, for $|G| / n_{2}<60$, we have $|G| / n_{2}=p, p^{2}, p q, 12$ or 56, where $p$ and $q$ are primes with $p \mid(q-1)$.

(f) If $|G| / n_{2}=p^{2}, p q, 12$ or 56 , where $p$ and $q$ are primes with $p \mid(q-1)$, then $|G| / n_{3}=p, 3$ or 7 .

Let $H(G)=k$, a positive integer. Note that $k=3$ or 4 , since $H(G)<\tau(G)$ and $G \nRightarrow C_{6}$.

First we consider the case $k=3$. In this case, by the definition of $H(G)$, we have

$$
2|G|=3\left(1+n_{1}+n_{2}+n_{3}\right) .
$$

Therefore, in view of the above assertions, it follows that $n_{3}=|G| / 2$ and $n_{2}=|G| / 10$. Hence, using (4), we have $|G|=60$ or 90 .

Next we consider the case $k=4$. In this case, by the definition of $H(G)$, we have

$$
|G|=4\left(1+n_{1}+n_{2}+n_{3}\right) .
$$


Therefore, in view of the above assertions, it follows that $n_{3}=|G| / 5$ and $n_{2}=|G| / 25$.

In case of (A) and (B), we also have $n_{1} \mid n_{2}$. Therefore, using (5) and the above assertions, it follows that $n_{1}=2,4$ and $|G|=300$ or 500 .

In case of (C), we have $n_{3}=n_{1} n_{2}$. Therefore, it follows that $n_{1}=5$, and hence, by (5), we have $|G|=600$. The contradictions to the choice of $G$ obtained at various stages prove the proposition.

Summarizing the above results we get the following Theorem.

THEOREM 4.5. If $G \neq C_{6}$ is a non-trivial harmonic group, then $\tau(G) \geq 6$.

\section{Harmonic groups of order pqr}

We have seen that a group $G$ of order $p q$, where $p<q$ are primes, is harmonic if and only if $G \cong C_{6}$. In this section, we characterize all harmonic groups of order $p q r$, where $p<q<r$ are primes. However, we begin with the following elementary lemma.

LEMMA 5.1. Let $G$ be a finite group such that $|G|$ is square-free. Then no two normal subgroups of $G$ have the same order. Consequently, $\tau(G) \leq 2^{\omega}$, where $\omega$ is the number of distinct prime factors in $|G|$.

Proof. It is enough to note that if $M, N \unlhd G$ such that $|M|=|N|$ but $M \neq N$, then $M N \unlhd G$ but $|M N|=|M||N| /|M \cap N|=|M|^{2} /|M \cap N|$ is not square-free as $M \cap N \neq M$. The consequential part is clear because for each positive divisor $d$ of $|G|$ there is at most one normal subgroup of $G$ order $d$.

The main result of this section is as follows.

Proposition 5.2. Let $G$ be a finite group with $|G|=p q r$, where $p<q<r$ are primes. Then $G$ is harmonic if and only if $G \cong C_{5} \times S_{3}$.

Proof. It is easy to see that $C_{5} \times S_{3}$ is harmonic; in fact, $H\left(C_{5} \times S_{3}\right)=$ $H\left(C_{5}\right) H\left(S_{3}\right)=(5 / 3)(9 / 5)=3$.

Conversely, suppose that $G$ is a harmonic group with $|G|=p q r$ and $H(G)=k$, where $p<q<r$ are primes and $k$ is a positive integer. Note that 
$2<k<\tau(G)$, and

$$
|G|(\tau(G)-k)=k(\sigma(G)-|G|) .
$$

By Theorem 4.5 and Lemma 5.1, we have $6 \leq \tau(G) \leq 8$. In fact, $\tau(G)=6$; otherwise, $G$ is a cyclic group, which is impossible as $C_{6}$ is the only abelian harmonic group whose order is square-free. Again, it is easy to see that $G$ always has unique normal subgroups of order $1, r, q r$ and $p q r$; moreover, considering the intersection and the product of normal subgroups, one can also notice that the remaining two normal subgroups of $G$ are of order $q$ and $p r, p$ and $p r$, or $q$ and $p q$. Thus, the possible values of $\sigma(G)$ are $1+q+r+p r+q r+p q r, 1+p+r+p r+q r+p q r$ and $1+q+r+p q+q r+p q r$. For the first two values of $\sigma(G)$, it follows from (6) that $r \mid k$, and so, we have $r=k=5$ as $2<k<6$ and $r \geq 5$; consequently, $q=3$ and $p=2$. However, using (6), one can easily see that these values of $k, p, q$ and $r$ are not consistent. Therefore, we have $\sigma(G)=1+q+r+p q+q r+p q r$. Now, it follows from (6) that for $k=5$ one of $p, q$ and $r$ equals 5 , whereas, for $k=4$ we have $p=2$. But the inconsistency of these values can be easily ascertained invoking (6) once again. Hence, we have $k=3$. Now, by (6), we have

$$
\begin{aligned}
& (1+q)(1+r)=p q(r-1) \geq 2 q(r-1), \quad \text { since } p \geq 2 \\
\Longrightarrow & 1+3 q \geq r(q-1) \geq q^{2}-1, \quad \text { since } r \geq q+1 \\
\Longrightarrow & 2 \geq q(q-3) .
\end{aligned}
$$

It follows that $q=3$, and so, $p=2$; consequently, by (6), we also have $r=5$. Thus, $|G|=30$. Since $G$ has a normal subgroup of order 5 as well as of order 6 , and also since $G$ is obviously non-abelian, it is a routine matter to see that $G \cong C_{5} \times S_{3}$. This completes the proof.

Acknowledgment. The authors are grateful to the referee for a number of useful suggestions.

\section{REFERENCES}

[1] G. L. CoHen, Numbers whose positive divisors have small integral harmonic mean, Math. Comp., 66 (1997), pp. 883-891.

[2] A. K. DAS, On arithmetic functions of finite groups, Bull. Austral. Math. Soc., 75 (2007), pp. 45-58.

[3] M. Garcia, On numbers with integral harmonic mean, Amer. Math. Monthly, 61 (1954), pp. 89-96. 
[4] T. Goto - S. Shibata, All numbers whose positive divisors have integral harmonic mean up to 300, Math. Comp., 73 (2004), pp. 475-491.

[5] T. Gото - K. OkeYA, All harmonic numbers less than $10^{14}$, Japan J. Indust. Appl. Math., 24 (2007), pp. 275-288.

[6] H. J. KANOLD, Über das harmonische Mittel der Teiler einer natürlichen Zahl, Math. Ann., 133 (1957), pp. 371-374.

[7] H. Kurzweil - B. Stellmacher, The theory of finite groups, SpringerVerlag, New York, 2004.

[8] T. LeInster, Perfect numbers and groups, arXiv:math. GR/0104012v1Apr2001.

[9] T. DE MEDTS - M. TăRnăUCEANU, Finite groups determined by an inequality of the orders of their subgroups, Bull. Belg. Math. Soc. Simon Stevin, 15 (4) (2008), pp. 699-704.

[10] T. De Medts - A. Maróti, Perfect numbers and finite groups, Rend. Sem. Mat. Univ. Padova, 129 (2013), pp. 17-33.

[11] O. ORe, On the Averages of the Divisors of a Number, Amer. Math. Monthly, 55 (1948), pp. 615-619.

[12] M. W. ShoRT, The Primitive soluble permutation groups of degree less than 256, Lect. Notes in Maths. 1519, Springer-Verlag, Berlin, 1992.

[13] M. TĂRnĂUCEANU, Finite groups determined by an inequality of the orders of their normal subgroups, Sci. An. Univ. “Al.I. Cuza” Iasi, Math., 57 (2011) pp. 229-238.

[14] MathOverflow, http://mathoverflow.net/questions/54851.

[15] The GAP Group, GAP - Groups, Algorithms, and Programming, Version 4.6.4, 2013 (http://www.gap-system.org).

Manoscritto pervenuto in redazione l'8 luglio 2013. 
